\title{
TESTING OF HYBRID NICKEL-POLYURETHANE FOAMS AT HIGH STRAIN-RATES USING HOPKINSON BAR AND DIGITAL IMAGE CORRELATION
}

\author{
Marcel Adorna ${ }^{a, *}$, Petr Zlámal ${ }^{a}$, Tomáš Fíla $^{a}$, Jan Falta $^{a}$, \\ Markus Felten ${ }^{b}$, Michael Fries ${ }^{b}$, Anne $\mathrm{Jung}^{b}$ \\ ${ }^{a}$ Czech Technical University in Prague, Faculty of Transportation Sciences, Department of Mechanics and \\ Materials, Konviktská 20, 12000 Prague 1, Czech Republic \\ ${ }^{b}$ Universität des Saarlandes, Institute of Applied Mechanics, Campus A4.2, Saarbrücken, 66123, Germany \\ * corresponding author: adorna@fd.cvut.cz
}

\begin{abstract}
In this paper Split Hopkinson pressure bar (SHPB) was used for dynamic testing of nickel coated polyurethane hybrid foams. The foams were manufactured by electrodeposition of a nickel coating on the standard open-cell polyurethane foam. High strength aluminium alloy bars instrumented with foil strain-gauges were used for dynamic loading of the specimens. Experiments were observed using a high-speed camera with frame-rate set to approx. 100-150 kfps. Precise synchronisation of the high-speed camera and the strain-gauge record was achieved using a through-beam photoelectric sensor. Dynamic equilibrium in the specimen was achieved in all measurements. Digital image correlation technique (DIC) was used to evaluate in-plane displacements and deformations of the samples. Specimens of two different dimensions were tested to investigate the collapse of the foam structure under high-speed loading at the specific strain-rate and strain.
\end{abstract}

KEYWORDS: SHPB, hybrid foam, DIC, strain-gauge measurement.

\section{INTRODUCTION}

In recent years, we have seen increasing interest in the use of light metal foams in the automotive, aerospace and other applications. This is mainly due to weight reduction, while maintaining load and strength of the construction. Another advantage is the ability to achieve better energy absorption capabilities and suitable acoustic damping properties [1]. For design and optimization in the aforementioned applications detailed description of the material properties, particularly under dynamic loads, is necessary. Metal foams and similar soft materials in general exhibit a nonstandard deformation behaviour in comparison with conventional materials (e. g. metals) 2, making it difficult to adopt standard measurement techniques for their high strain-rate characteristics and complex deformation behaviour 3 6. This work is focused on the investigation of the deformation behaviour of nickel coated polyurethane hybrid foam using an innovative SHPB device in combination with DIC. The presented experimental data describes the course of the foam structure collapse and extends the knowledge of deformation mechanism of hybrid nickel-polyurethane foams, where a coating of nano-crystalline nickel is deposited on cheap open-cell polyurethane foams.

\section{MATERIALS AND METHODS}

\subsection{SAMPLE PREPARATION}

Polyurethane (PU) foams with a pore size $20 \mathrm{ppi}$ (pores per inch) from Jumpinoo, Spenge, Germany was used. Two types of cylindrical specimens with diameter of approx. $20 \mathrm{~mm}$ and height approx. $20 \mathrm{~mm}$ (long specimens) and approx. $10 \mathrm{~mm}$ (short specimens) were cut by hot wire cutting from the foam plates. Since PU foams are not electrically conductive, the foams must be rendered electrically conductive by dip coating in a graphite lacquer (CRC Kontakt Chemie, Iffezheim, Germany) to be able to coat the foams by electrodeposition. The electrodepostion of nanocrystalline nickel (Ni) on the carbon coated PU foams was carried out using a commercial Ni sulfamate electrolyte with a $\mathrm{Ni}$ content of $110 \mathrm{~g} / \mathrm{l}$ (Enthone GmbH, Langenfeld, Germany) at an electrolyte temperature $50{ }^{\circ} \mathrm{C}$ and $\mathrm{pH}$ 3.8. In order to guarantee for a nearly homogeneous coating thickness all over in the foam, the foam was centered as cathode in a double-walled cube filled with Ni pellets (A.M.P.E.R.E. GmbH, Dietzenbach, Germany) acting as sacrificial anode. The electroplating was performed at current density $1.5 \mathrm{~mA} / \mathrm{cm}^{3}$ until a theoretical coating thickness of $75 \mu \mathrm{m} \mathrm{Ni}$ was deposited. For more details, it is referred to the literature [7 9. The specimen of hybrid foam in the loading device is shown in Fig. 1 .

\subsection{Digital image CORRELATion}

The deformation of the sample during the test was recorded using a high-speed camera. High-speed imaging enables to capture the deformation process of SHPB tests in suitable quality (sufficient number of images with small changes between them) for the strain assessment. Based on the captured images, changes 


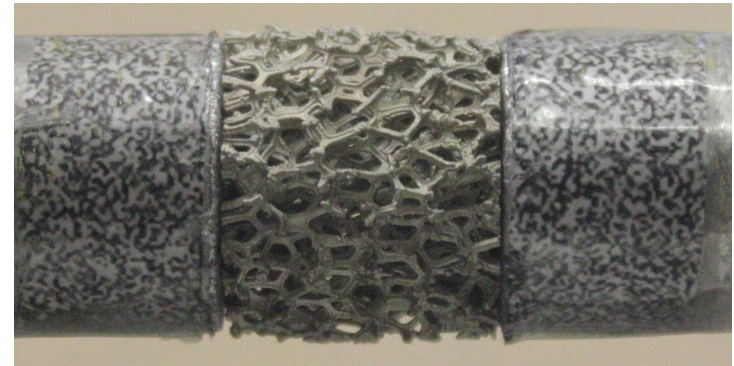

Figure 1. Ni/PU foam specimen in the SHPB experimental setup

of the sample structure can be evaluated, using digital image correlation (DIC) technique. Nowadays, DIC technique is a well established method for the assessment of strain in the complex structure using tracking of virtual pattern selected in the sequence of images where the displacements are to be evaluated. Each point of the virtual pattern is subsequently tracked through to each pair of images (reference and deformed) in the series of loading states. The computation implementation of the tracking algorithm is based on subsets of pixels which are defined as a square shaped area with defined pixel dimensions formed around the individual tracked point (Fig. 2). The size of the subset area has to be suitably chosen with respect to computational cost and traceability. As the specimen is deformed, the reference square pattern is also deformed (combination of translational, rotational and shear deformations) in the image sequence. The position of the deformed subset in the image is calculated based on the extreme of correlation coefficient calculated using the selected criterion. In this work, the algorithm derived from the LucasKanade tracking procedure [10 and implemented in a custom DIC tool and the matlab toolkit [11] was used. In this tool, the correlation coefficients were estimated during the two-step procedure, first at pixel level and second at sub-pixel level to determine the values of displacement more accurately. At the pixel level, the sum-squared difference (SSD) criterion was used whereas the Lucas-Kanade algorithm based on the zero-normalised SSD (ZNSSD) criterion was used at the sub-pixel level. Then, strain fields could be calculated from resulting displacements in two dimensions of each point (output of DIC algorithm) and mapped to the original structure to describe the inplane deformation behaviour of the sample.

\subsection{Split Hopkinson Pressure Bar}

Testing of the hybrid foams was performed using a modified Kolsky SHPB setup. Diameter of the measurement bars and the striker bar was $20 \mathrm{~mm}$. All bars were made of high-strength aluminium alloy (ENAW-7075). The striker was propelled using a gas-gun with 16 bar maximum pressure. The gas-gun system was composed of the following parts: a steel barrel with the maximum stroke $2500 \mathrm{~mm}$, a high-flow fast

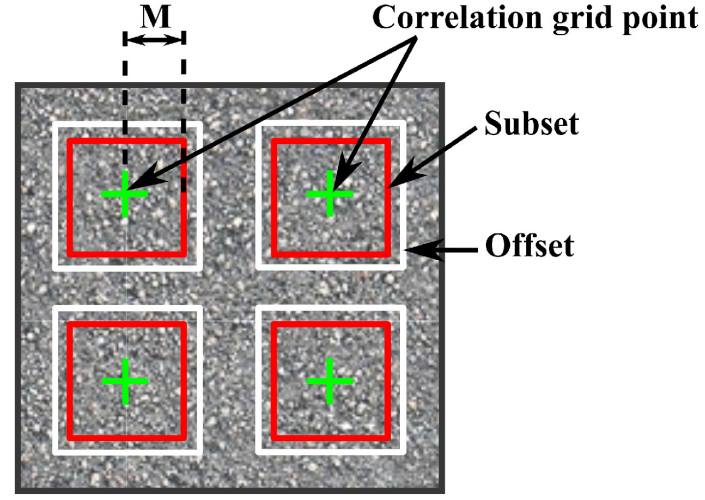

Figure 2. Parameters of the Digital Image Correlation points, parameter $\mathrm{M}$ represents the dimension of subset area

release solenoid valve (366531, Parker, USA), a 201 air reservoir equipped with pressure gauge and other peripherals (compressor, piping etc.). Length of the both incident and transmission bar was $1600 \mathrm{~mm}$. The bars were supported by a set of a low friction polymerliner slide bearings (Drylin FJUM, IGUS, Germany) mounted in a custom stainless steel housing. The striker bar had length $500 \mathrm{~mm}$. The surfaces of the bars were precisely finished to maintain a tight diameter tolerance for smooth motion in the bearings without notable clearance. The axis of the bars was carefully set to minimize friction and to maintain straightness of the SHPB setup. Geometrical alignment is very important for the minimization of the undesirable effects (increased friction, bending of the bars etc.) affecting the measurement accuracy. The impact faces of the bars were precisely ground and were set to maintain plan-parallelism between the faces better than $0.05 \mathrm{~mm}$ (measured by feeler gauge). An overview of the experimental setup is shown in Fig. 3

\subsection{INSTRUMENTATION}

The strain wave passing through the incident and transmission bar was measured at two measurement points (MPs) using foil strain gauges (3/120 LY61, HBM, Germany) with $3 \mathrm{~mm}$ active length. The strain gauges were arranged to Wheatstone half-bridge circuit to double the output signal in comparison to single gauge and to eliminate the potential influence of bending of the bars. On each measuring bar $(1600 \mathrm{~mm}$ in length) one MP was set at a distance of $800 \mathrm{~mm}$ from each bar end. The foil strain gauges were selected for this application due to their linearity and range of possible strain measurement (up to $50,000 \mu \varepsilon$ ). The disadvantage of foil gauges is the need to amplify the signal before digitization. This disadvantage can be solved by using semiconductor strain gauges but their nonlinear characteristic and low measurement range (up to $2,500 \mu \varepsilon$ ) makes them unsuitable for our application. Each strain gauge was bonded on the bar 


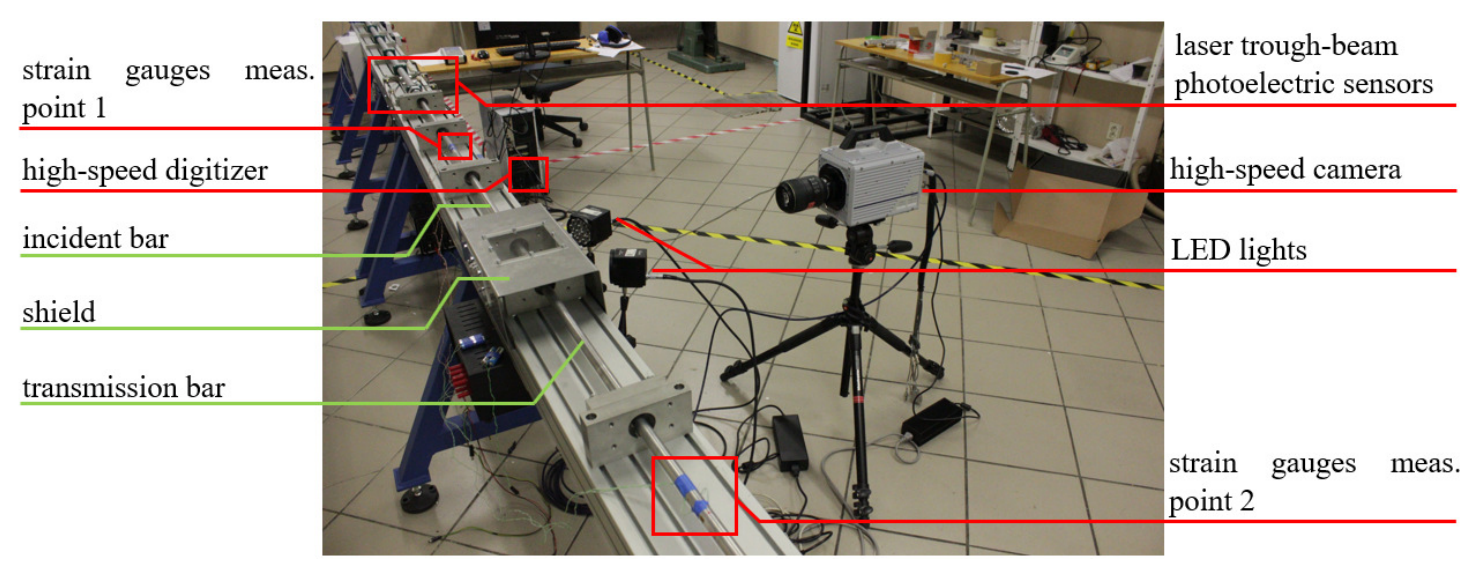

FiguRE 3. SHPB experimental setup overview

with a single component low-viscosity cyanoacrylate adhesive (Z70, HBM, Germany) and cured for at least 12 hours to ensure maximum strength of the bond. To reduce the noise in the measurement chain (maximize to signal-to-noise ratio of the output signal) the powering of the MPs was performed using custom battery units with an excitation voltage $4 \mathrm{~V}$. MPs signal output were amplified using active differential low noise amplifier (EL-LNA-2, Elsys AG, Switzerland) with gain 100 and sampled using high speed 16-bit digitizers (PCI-9826H, ADLINK Technology, Inc., Taiwan) with a maximal $20 \mathrm{MHz}$ sample rate and triggered by signal of through-beam photoelectric sensor (FS/FE 10-RL-PS-E4, Sensopart, Germany) installed on the barrel of the SHPB. The signal from the through-beam photoelectric sensor is also used for the high-speed camera triggering and time synchronization of the strain gauges signal with the captured image sequence. For accurate DIC evaluation it is crucial to capture the images in sufficient quality, i.e, properly illuminated and with satisfactory frame rate to ensure a sufficiently small structure changes between the images. Thus, the deformation of the sample during the SHPB test was observed using a high-speed camera (FASTCAM SA5, Photron, Japan) with a selected region of interest (RIO) with pixel resolution $256 \times 216$. The selected ROI enabled to reach a frame rate of approximately $105 \mathrm{kfps}$ and 4 pixels resolution per strut thickness of hybrid foam. Illumination of the sample surface was performed using a pair of high intensity LED lights (Constellation 60, Veritas, USA). Data acquisition was employed using a custom virtual instrument designed in Labview environment (National Instruments, USA) with TDMS binary file output format also readable in the matlab toolkit for further analysis.

\section{Results}

\subsection{Digital image CORRElation Results}

Two sets of specimen with nominal diameter $20 \mathrm{~mm}$ and nominal length $10 \mathrm{~mm}$ (short samples) and $20 \mathrm{~mm}$ (long samples) were measured using SHPB at two dif- ferent strain-rates. In the paper, the individual strainrates are designated low and high. This designation is relative to the sample. Strain-rate achieved during experiments depends not only on striker velocity, but also lenght of tested sample (short samples - low strain rate ca. $1500 s^{-1}$, high strain rate ca. $5000 s^{-1}$, long samples - low strain rate ca. $1400 s^{-1}$, high strain rate ca. $2200 s^{-1}$ ).

An identical grid of $22 \times 14$ correlation points was used to cover the area of the specimen and the end parts of the measuring bars on the high-speed camera images. The position of the grid points was tracked using DIC and new positions of the grid were identified in the whole sequence of the captured high-speed camera images which were converted from raw format to 8-bit png by a lossless compression algorithm and were not subjected to any additional preprocessing. In all cases, digital image correlation was successful, the mean correlation coefficient was higher than $90 \%$ for all measurements. In Fig. 4 median value of the correlation coefficient for three representative experiments: short sample at low strain-rate (test 270), short sample at high strain-rate (test 285), long sample at high strain-rate (test 317 ) is plotted against strain evaluated using DIC. It demonstrates that the trend of all three curves is the same and that the DIC results for short samples can be considered reliable up to approx. 0.3 overall strain. In this strain range, the strains evaluated using DIC are in very good agreement with the strain-gage measurements. In case of the long samples, the median correlation coefficient exhibits similar trend with dramatic drop of its value for strain higher than 0.2 .

In strain higher than $0.2-0.3$, the results provided by DIC started to be distorted and unreliable. This phenomena is represented by the drop and the oscillations of the correlation coefficient curves (see green and blue curve in Fig. 4) and significant differences in stress-strain curves evaluated using DIC and straingauge at strain higher than $0.2-0.3$ (see Fig. 6-77). Evolution of the full-field in-plane strain of the foam structure evaluated using DIC is shown in Fig. 8 for three selected representative cases. Loss of correlation 
was caused by the densification of the foam structure changing significantly the original structure of the foam. Another reason was that diameter of the specimen during compression started to be higher than the diameter of the bars. Strain was therefore localized in the central part of the specimen. This distortion caused by the increase of the diameter was even more significant for longer samples (Fig. 8 bottom). The dynamic equilirium was achieved in all experiments as the samples were deformed uniformly on both ends in short time after the wave impact on the specimen(see Fig. 8- second column of the images). In Fig. 8 it is also shown, that hybrid foam deformation occurs locally in areas where the structure is the weakest and collapse of the structure occurs on this plane.

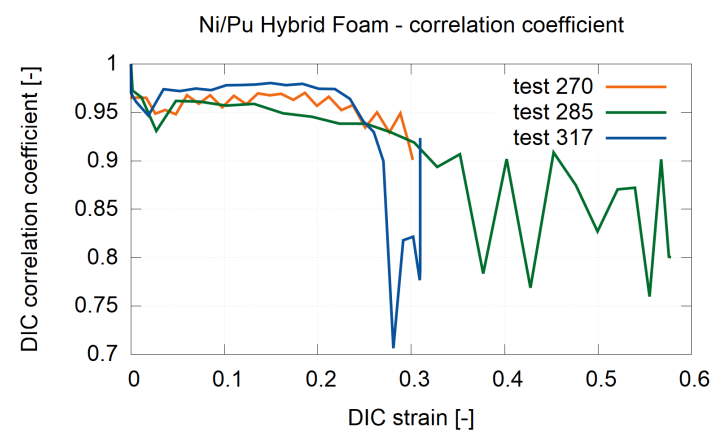

FiguRE 4. Representative median value of the correlation coefficient plotted against strain for three selected experiments (test 270 - short sample at low strain rate, test 285 - short sample at high strain rate, test 317 long sample at high strain rate)

\subsection{DIC AND STRAIN-GAUGE MEASUREMENT RESULTS COMPARISON}

Stress-strain curves with strain derived using DIC were evaluated for each tested sample and compared with the strain-gauge curves. A comparison for three measurements (corresponding with full-field DIC analysis shown in Fig. 8) are shown in Fig. 5 to Fig. 7 (green lines used for DIC curve, orange lines for strain-gauge results). In all cases, stress-strain curves with strain derived using DIC are very close to the strain-gauge curves up to 0.3 strain for the short specimens and 0.2 for the long specimens. Differences of the DIC curves to strain-gauge curves after 20-30\% deformation are caused by the fact that sample size exceeds the measuring bars diameter, resulting in a loss of correlation points in parts outside the monitored area. Moreover, significant deformation of the sample also leads to a loss of correlation points due to the collapse of the structure and shift of individual struts out of high-speed camera focus.

\section{Conclusion}

SHPB experimental device was successfully used for the dynamic compression of the $\mathrm{Ni} / \mathrm{PU}$ hybrid foam. In-plane strain was successfully evaluated from the

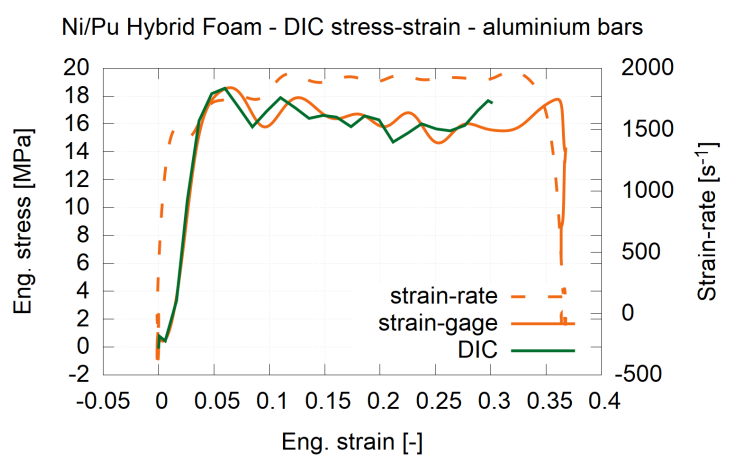

FiguRE 5. Comparison of stress-strain diagrams with strain evaluated using strain-gage and DIC, experiment no. 270

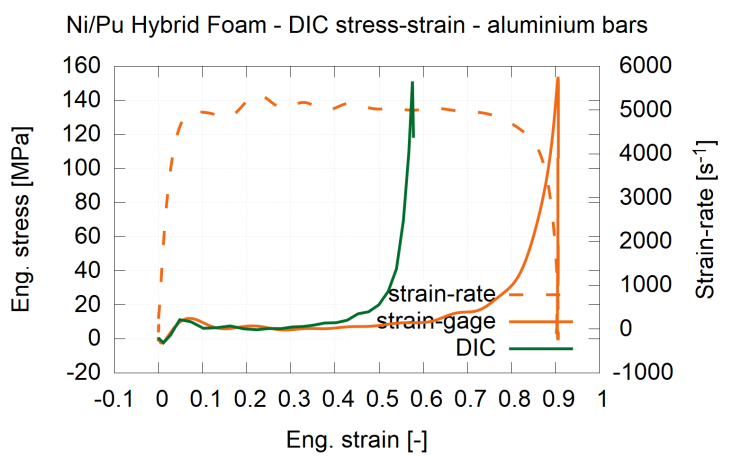

Figure 6. Comparison of stress-strain diagrams with strain evaluated using strain-gage and DIC, experiment no. 285

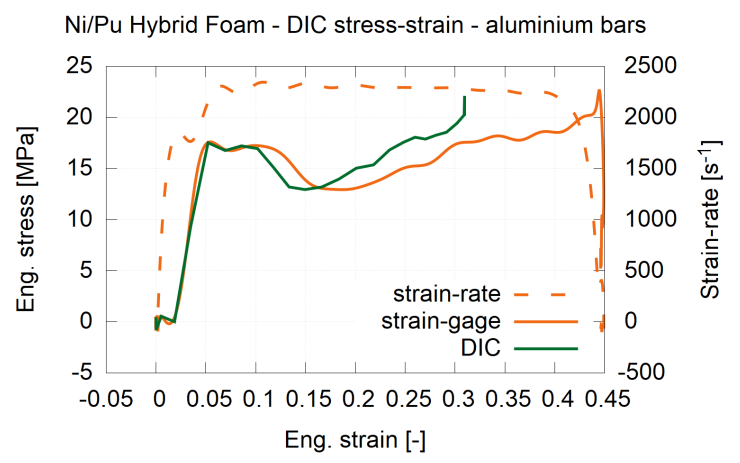

Figure 7. Comparison of stress-strain diagrams with strain evaluated using strain-gage and DIC, experiment no. 317

high-speed camera images using digital image correlation technique. DIC results were found to be in a very good agreement with strain-gauge measurements up to $30 \%$ of deformation for the short samples and $20 \%$ of deformation for the long samples. Dynamic equilibrium was achieved in all measurements as the specimens were deformed uniformly on both ends in short time after the wave impact on the specimen. It was shown that both SHPB and DIC are suitable tools for advanced characterization of the hybrid foam under impact loading. 


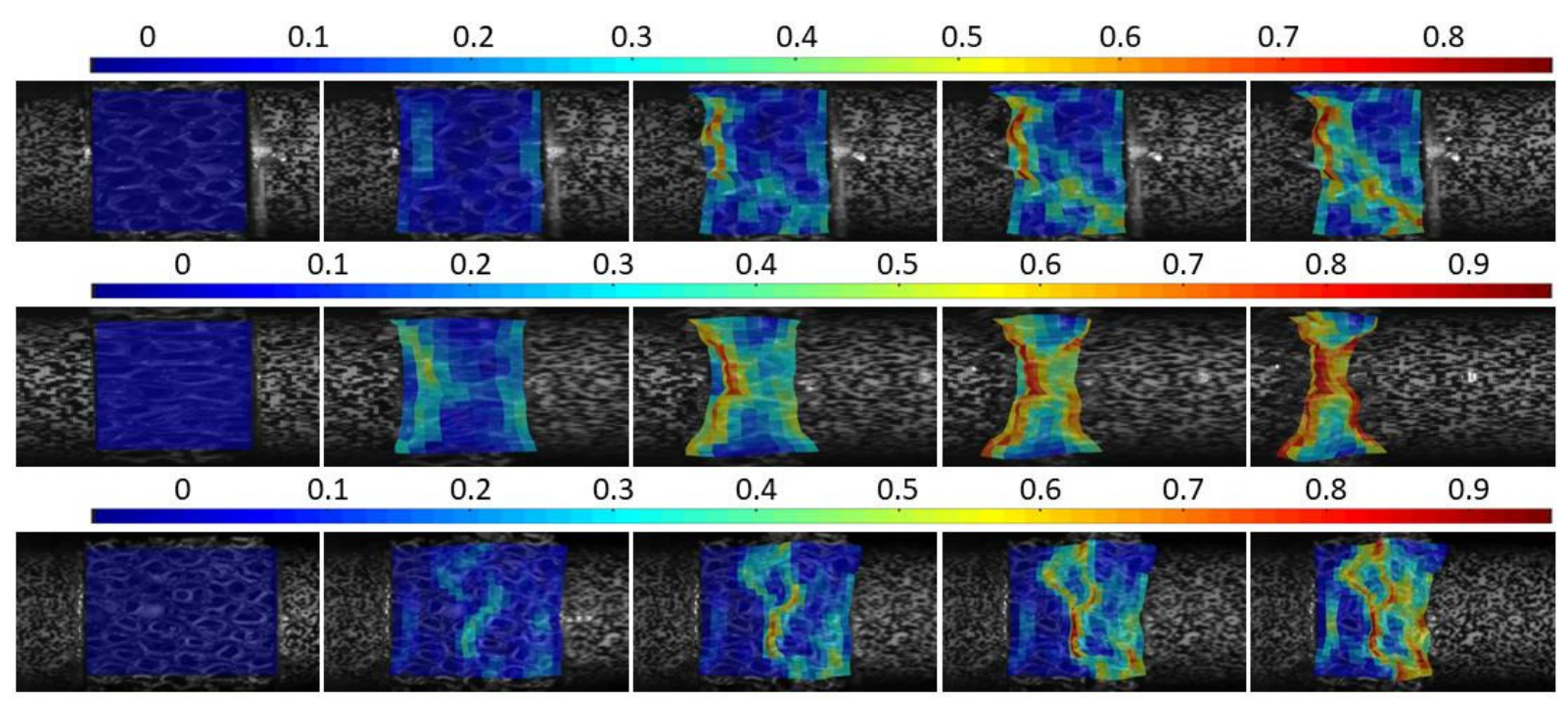

FiguRE 8. Full-field DIC analysis: in-plane longitudinal strain

Top row: Experiment no. 270, $v_{s}=22.1 \mathrm{~ms}^{-1}$ (low strain-rate, short sample), $d=22.4 \mathrm{~mm}, l=10.1 \mathrm{~mm}$

Middle row: Experiment no. 285, $v_{s}=38.1 \mathrm{~ms}^{-1}$ (high strain-rate, short sample), $d=21.9 \mathrm{~mm}, l=7.5 \mathrm{~mm}$ Bottom row: Experiment no. 317, $v_{s}=43.7 \mathrm{~ms}^{-1}$ (high strain-rate, long sample), $d=21.8 \mathrm{~mm}, l=18.6 \mathrm{~mm}$ $v_{\mathrm{s}}\left[\mathrm{ms}^{-1}\right]$ - Striker velocity, $d[\mathrm{~mm}]$ - Sample diameter, $l[\mathrm{~mm}]$ - Sample length

\section{ACKNOWLEDGEMENTS}

The research was supported by the Czech Science Foundation (project no. 15-15480S) and the internal grants of the Czech Technical University in Prague (projects no. SGS17/148/OHK2/2T/16 and no. SGS18/153/OHK2/2T/16)). All the financial support is gratefully acknowledged.

\section{REFERENCES}

[1] T. Fila, P. Zlamal, O. Jirousek, et al. Impact testing of polymer-filled auxetics using split hopkinson pressure bar. Advanced Engineering Materials 19(10), 2017. DOI:10.1002/adem.201700076.

[2] G. Sunny, F. Yuan, V. Prakash, J. Lewandowski. Design of inserts for split-hopkinson pressure bar testing of low strain-to-failure materials. Experimental Mechanics 49(4):479-490, 2009. DOI:10.1007/s11340-008-9145-1.

[3] P. Wang, S. Xu, Z. Li, et al. Experimental investigation on the strain-rate effect and inertia effect of closed-cell aluminum foam subjected to dynamic loading. Materials Science and Engineering A 620:253-261, 2014. DOI:10.1016/j.msea.2014.10.026.

[4] A. Jung, M. Larcher, O. Jirousek, et al. Strain-rate dependence for ni/al hybrid foams. vol. 94. 2015. DOI:10.1051/epjconf/20159404030.

[5] K. Dannemann, J. Lankford Jr. High strain rate compression of closed-cell aluminum foams. Materials Science and Engineering A 293(1):157-164, 2000. DOI:10.1016/S0921-5093(00)01219-3
[6] V. Deshpande, N. Fleck. High strain rate compressive behaviour of aluminum alloy foams. International Journal of Impact Engineering 24(3):277-298, 2000. DOI:10.1016/S0734-743X(99)00153-0

[7] A. Jung, H. Natter, S. Diebels, et al. Nanonickel coated aluminum foam for enhanced impact energy absorption. Advanced Engineering Materials 13(1-2):23-28, 2011. DOI:10.1002/adem.201000190

[8] A. Jung, K. M. R., E. Lach, et al. Hybrid metal foams: Mechanical testing and determination of mass flow limitations during electroplating. International Journal of Material Science 2(4):97-107, 2012. DOI:10.1002/adem.201000190

[9] A. Jung, S. Diebels. Synthesis and mechanical properties of novel Ni/PU hybrid foams: A new economic composite material for energy absorbers. Advanced Engineering Materials 18(4):532-541, 2015. DOI:10.1002/adem.201500405

[10] B. D. Lucas, T. Kanade. An iterative image registration technique with an application to stereo vision. In Proceedings of Imaging Understanding Workshop, pp. 674-679. 1981.

[11] I. Jandejsek, J. Valach, D. Vavrik. Optimization and calibration of digital image correlation method. pp. 131-136. 2010. 